\title{
Evaluation of muscle fatigue using infrared thermal imaging technique with assisted electromyography
}

\author{
Nursyazana Ridzuan a, Aizreena Azaman b,c, Kamaruzaman Soeed c , Izwyn Zulkapri a, Asnida \\ Abdul Wahab a, ${ }^{*}$ \\ a Department of Clinical Sciences, Faculty of Biosciences and Medical Engineering, 81310 UTM Johor Bahru, Johor, Malaysia \\ ${ }^{b}$ Sport Innovation Technology Center , 81310 UTM Johor Bahru, Johor, Malaysia \\ ${ }^{c}$ Department of Biotechnology and Medical Engineering, Faculty of Biosciences and Medical Engineering, 81310 UTM Johor Bahru, Johor, \\ Malaysia \\ *Corresponding author: asnida@biomedical.utm.my
}

\section{Article history}

Received 15 October 2017

Accepted 6 December 2017

\begin{abstract}
Muscle fatigue in sports science is an established research area where various techniques and types of muscles have been studied in order to understand the fatigue condition. It can be used as an indicator for predicting muscle injury and other muscle problems which can decrease athletes' performance. Muscle fatigue usually occurs after a long lasting or repeated muscular activity. Electromyography (EMG) assessment method is a standard tool used to evaluate muscle fatigue based on the signals from the neuromuscular activation during fatigue condition. However, additional time for equipment set up such as placement of the electrodes and the use of multiple wires make this overall setting a bit complicated. In addition, the signal from EMG which possessed some noise, need to be filtered and post processing time is also required to obtain a reliable measurement signal. Therefore, researchers have explored the application of thermal imaging technique as one of the alternative methods for muscle fatigue assessment. The objective of this study is to investigate the correlation of muscle fatigue condition measured using a non-invasive infrared thermal imaging technique and a standard evaluation method, EMG. Five healthy men were selected to run on a treadmill for 30 minutes with a constant speed setting. Temperature and EMG signals were registered from gastrocnemius muscle of the subjects' dominant leg simultaneously. Result obtained shows that the average temperature of gastrocnemius muscle decrease as subjects start to exercise. Further temperature decrease along with exercise and increase in temperature were observed during the recovery period. Statistical analysis was performed and analyzed using both temperature and EMG parameters. Result shows a significant strong correlation with $r=0.7707$ and $p<0.05$ between temperature difference and median frequency (MDF) for all subjects compared to average temperature. Therefore, it is concluded that temperature difference extracted from thermal images can be used as an ideal parameter for muscle fatigue evaluation.
\end{abstract}

Keywords: Muscle fatigue, thermal imaging, muscle injury, thermoregulation

\section{INTRODUCTION}

Muscle fatigue in sports science is a condition when there is a transient decrease in contractile strength and capacity to perform physical actions. Muscle fatigue occurs after a long lasting or repeated muscular activity. During this phase, the capacity of muscle to produce maximum voluntary action or to perform a series of repetitive actions is reduced (Hadžić et al., in press). For athletes, muscle fatigue plays a crucial role since it limits their performance in sport. Muscle fatigue can be used as a good indicator to prevent a person from having other musculoskeletal diseases (MSD) such as muscle injury and thus, it is important to assess muscle fatigue condition in its early phase (AlMulla et al., 2011).

According to Bartuzi et al. (2012) the most common and leading method since 1980s in assessing muscle fatigue in sports science is by using electromyography (EMG). This method is used to estimate fatigue by quantifying electrical signals sent to muscle fibers through motor neurons during muscle activation. EMG is a diagnostic procedure to assess muscle health and the nerve cells that control the muscle (motor neurons). This motor neurons transmit electrical signals that cause muscle to contract. EMG uses electrodes to transmit or detect electrical signals from the muscle. In accessing muscle load and fatigue during an occupational task, the electrical signal from a surface electrode is commonly used (Merletti et al., 2009).

Although EMG is the current preferred method for muscle fatigue, factors such as various sources of noise acquired with the signal such as electromagnetic noise, transducer noise, power line noise, and motion noise limit its performance (Al-Mulla et al., 2011). Moreover, measurement of EMG signal will require additional time for equipment set up and proper placement of the electrodes. In addition, post processing time is also needed for noise filtering in the signals. EMG also has the limitation in detection of low level of muscle contraction (Kallenberg and Hermens, 2008). Therefore, researchers have started to look for a reliable and better alternative method for muscle fatigue detection and prediction such as by using non-invasive infrared thermal imaging (Al-Mulla et al., 2011).

In any physical activities, heat is lost to the environment via convection and evaporation during respiration process. Based on the 
First Law of Thermodynamic a shown in Equation (1), the total of heat produced is equivalent to the difference between metabolic heat production and the rate of mechanical work (M-W) (Priego Quesada, 2017).

$$
M-W=(K+C+R+E)+S
$$

where $M$ represents the rate of metabolic heat production, $W$ is the rate of mechanical work, $K$ and $C$ represent the rate of conductive heat loss and the rate of convective heat loss from the skin respectively. $R$ is the rate of radiative heat loss from the skin, $E$ is the evaporative rate of heat loss from the skin, and finally $S$ is the rate of body heat storage

Cramer and Jay (2016) define the rate of conductive heat loss as "the rate of free energy released from the catabolism of carbohydrate, fat, and amino acids to resupply adenosine triphosphate (ATP) for cellular activities such as transport, biosynthesis, and muscular contractions" (Cramer and Jay, 2016). Some of this energy is converted in external work and the rest is converted into heat (Cramer and Jay, 2016). Since human body is inefficient in transforming the energy in mechanical work, $30-70 \%$ of the energy produced, transformed into thermal energy (Gonzàlez-Alonso, 2012).

Muscle fatigue can occur during exercise where muscles tend to become weak and tired and it will reduce the ability to contract and apply force after several times (Al-Mulla et al., 2011). Usually, this localized muscle fatigue will occur after a long duration or with a repeatable strong exercise muscle activity. There is no specific load and threshold which can be used to define this muscle fatigue as the muscle characteristics of each individual are inconsistent and varies. Muscle fatigue can help to promote muscle growth but it is usually harmful as high muscle fatigue level will cause subjects performance to decrease as well as increase in risk of serious injury (Hadžić et al., in press). Thus, it is crucial to understand the processes of human thermoregulation and its relation to skin temperature profile during exercise with the aim of obtaining the maximum possible performance and protecting athletes from injury, especially in extreme environments (Lim et al., 2008).

Recently, there are various applications in sports science which use infrared thermal imaging as monitoring and diagnostic tool such as sports medicine, analysis of thermoregulation during exercise (Arfaoui et al., 2012 ; Formenti et al., 2016), assessment of clothing in specific sport or during exercise (Gillis et al., 2016) sport equipment assessment (Fenner et al., 2016), and detection of overuse and traumatic knee injuries (Hildebrandt et al., 2012). Infrared thermal imaging is a noninvasive, non-radioactive, non-obstructive and contactless detection tool which is capable in assessing physiological functions related to changes in skin temperature. It can detect and localize the thermal changes which characterized by either the increase or decrease in skin temperature (Hildebrandt et al., 2012). Previous studies have shown that thermal imaging provides better results for athletes, as it is an instrument for identifying risks and preventing injuries, as well as an important tool for monitoring sports training, based on the evaluation of training load (Bartuzi et al., 2012 and Formenti et al., 2016).

However, current studies show that there is insufficient scientific evidence of its successful application in the clinical environment. Therefore, this study is performed to investigate the correlation of muscle fatigue condition by using infrared thermal imaging technique which assisted by a standard evaluation method, EMG.

A series of experiment was conducted to obtain both surface temperature and EMG signals from gastrocnemius muscle via a monitored running activity on the treadmill. A statistical analysis was then performed between both muscle temperature and EMG parameters in order to obtain the correlation between these methods. This study is beneficial for athletes and those who are involved in sports where the use non-invasive thermal imaging in accessing muscle fatigue is an effective alternative method compared to EMG due to its low cost and simple equipment setting. In addition, it is very useful in preventing any muscle injuries, implementing preventive strategies and thus improving their health and performance while reducing unnecessary rehabilitation cost.

\section{MATERIALS AND METHOD}

\section{Experimental Setup}

This study is an experimental study conducted in Fitness Gymnasium Laboratory, which located at Faculty of Biosciences and Medical Engineering (FBME), Universiti Teknologi Malaysia (UTM), Johor. Five healthy men from UTM, Johor have participated as the subjects for data collection purposes. This group was homogenous in terms of age, body weight and height as shown in Table (1).

Table 1 Subjects demographic distribution

\begin{tabular}{cc}
\hline Parameter (Unit) & Value \\
\hline Age ( year ) & $31 \pm 1.25$ \\
Body Weight ( kg ) & $76.8 \pm 3.11$ \\
Height $(\mathbf{c m})$ & $176.3 \pm 4.39$ \\
\hline
\end{tabular}

Based on the survey performed, all subjects are healthy and do not have any muscle injuries or any diseases. Subjects were required to read and signed the informed consent form prior to the study. Each subject was interviewed and the procedure was explained to them individually. In order to assess muscle fatigue in the targeted area of gastrocnemius muscle, subjects were required to run on the treadmill with the constant speed of $10 \mathrm{~km} / \mathrm{h}$ for 30 minutes. Measurement of surface temperature and EMG signal were taken from gastrocnemius muscle of the subjects' dominant leg as illustrated in Figure 1.

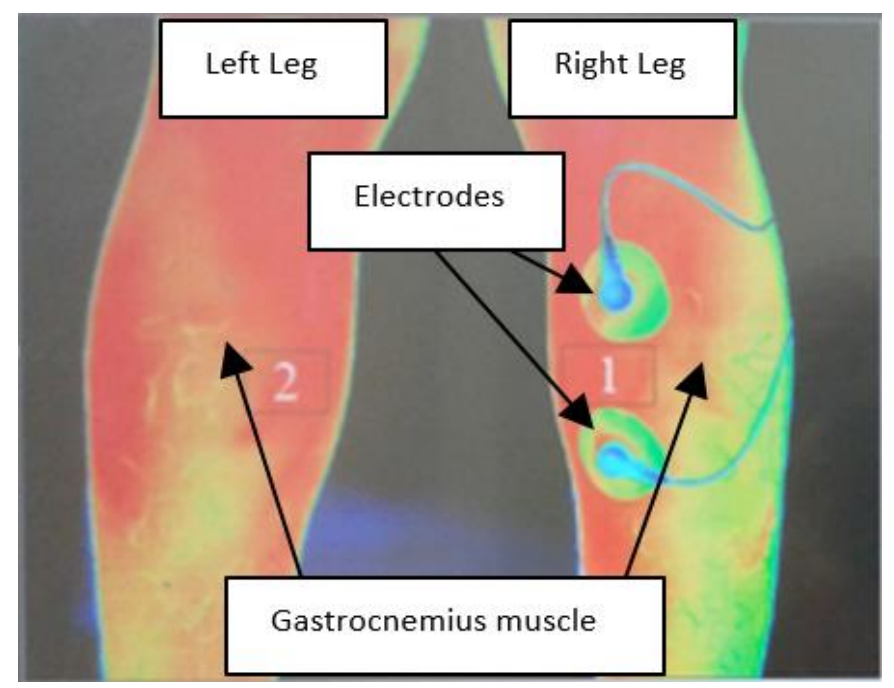

Fig. 1 Illustration of gastrocnemius muscle.

\section{Infrared Thermal imaging}

In order to ensure data validity, reliability and consistency, a standard protocol was followed accordingly for before, during and after the infrared thermal imaging measurement of skin surface temperature (Hildebrandt et al., 2012). Factors such as variations in room temperature, different percentages of light exposure, and other possible factors that could influence the result in a significant way were minimized. The room was prepared with a minimal light exposure and the room temperature was controlled and maintained at a range of $20^{\circ} \mathrm{C}$ to $22^{\circ} \mathrm{C}$, using air conditioning system in with relative humidity of 60 to $65 \%$. The fluorescent lights available in the room is switched off as much as possible during acclimatization and screening processes. A partition was also installed around the treadmill to reduce thermal reflection from the surrounding.

The images of surface temperature from gastrocnemius muscle were acquired using an Epidermal Thermal Imaging Professional (ETIP) infrared imaging camera system model 7640 P-Series, manufactured by Infrared Camera Incorporation, Texas USA. It can produce a thermal image with a resolution of $640 \times 480$ pixel, and a field of view of $49^{\circ} / 18 \mathrm{~mm} \times 36^{\circ} / 25 \mathrm{~mm}$, using a focal plane array microbolometer type detector, a spectral range of 7-14 $\mu \mathrm{m}$, a thermal sensitivity of $0.038^{\circ} \mathrm{C}$ with a temperature range of $-40^{\circ} \mathrm{C}$ to $400^{\circ} \mathrm{C}$ and 
an accuracy of $\pm 1 \%$ of readings. The camera was mounted on a flexible metal bar, and connected to the display monitor. The camera is adjusted to focus on the gastrocnemius muscle for both subject's leg. The distance of the camera to the targeted muscle was manually controlled, in order to get the best display output. The initialization of the camera was carried out prior to screening, in order to reduce noise and to stabilize the system.

Five thermal images were captured in a 5 seconds ( 1 frame/second) for each time sequence starting from static position where no running activity performed on the treadmill. As shown in Figure 2, the time sequence for this experiment was set to have an interval of 3 minutes during running on the treadmill until the first 20 minutes. Then thermal imaging images were then taken at the interval of 5 minutes until it reached 30 minutes. Lastly, thermal images of gastrocnemius muscle during the recovery state were taken at the interval of 3 minutes until it reached the duration of 48 minutes. All images were recorded using IR Flash Medical and stored in two different file formats namely JPEG and CSV formats. Various thermal parameters such as minimum temperature, maximum temperature and average temperature data were then extracted for further analysis.

\section{Electromyography}

The measurement of EMG signal was collected simultaneously with surface temperature using infrared thermal imaging. EMG signal was recorded at $2000 \mathrm{~Hz}$ for 30 minutes while subjects were running on the treadmill (Figure 2). The equipment used for EMG set up consist of EMG surface electrodes, and TMSi Porti system manufactured by Netherland. The Porti is a 32-channel ambulatory and stationary system for physiological research. It is a multifunctional system which have unipolar electrophysiological inputs (ExG), bipolar electrophysiological inputs (BIP) and auxiliary inputs (AUX) for measuring EMG. Before the surface electrodes being placed onto subjects' gastrocnemius muscle, skin preparation was performed where subjects were shaved at the targeted gastrocnemius muscle region and their skin surface were then wiped with an alcohol swab to remove the dead skin cells and any dirt that can produce high impedance. Then, two EMG surface electrodes were placed across the selected muscle fibers, gastrocnemius muscle of subjects' dominant leg and the third one was placed on the knee to acts as a reference or ground electrodes.

In order to get EMG results with minimum artifacts which give additional noise to the signals, the sources of artifacts such as electrical noise from power lines and external sources, motion artifacts, cross talk contamination, clipping and physiological noise need to be controlled as much as possible. The electrical noise is from the electronic equipment and electromagnetic radiation. While the sources of motion artifacts are from electrode interface and electrode cable that can be reduced by properly secured the wires to minimize motion. This will result in higher Signal to Noise (SNR) ratio. Median frequency (MDF) of the power spectrum was extracted to assess muscle fatigue in this study.

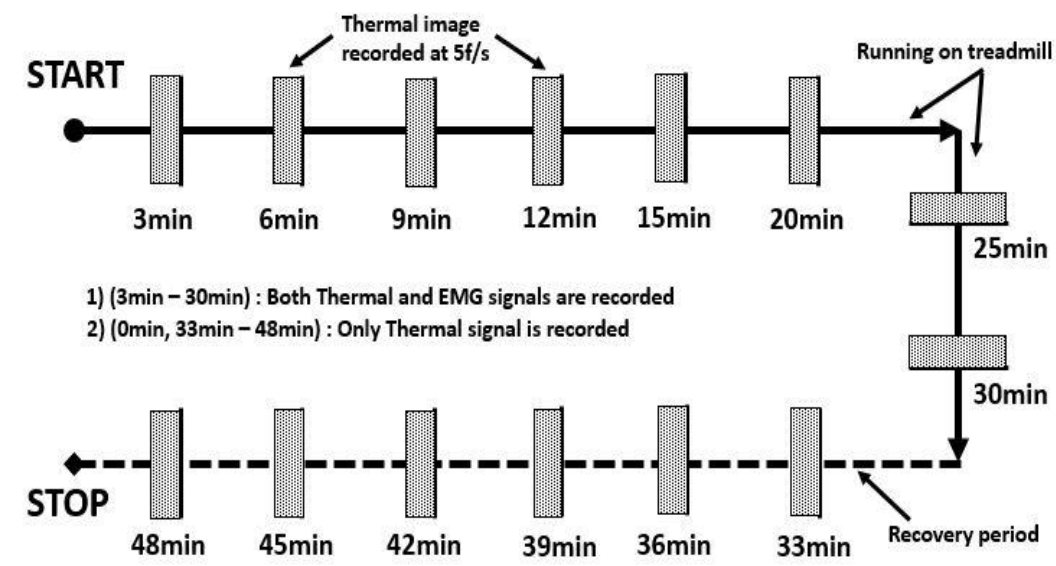

Fig. 2 Timeline for procedures in measuring surface EMG and Infrared Thermal imaging profiles.

\section{Data analysis}

All temperature reading parameters from thermal imaging camera were extracted from ETIP infrared imaging camera storage. The parameters involved are mean temperature, median temperature, average temperature and temperature difference for all subjects which were recorded before, during and after exercise. The data were then plotted in Microsoft Excel.

The signal of EMG was processes using Matlab software (MATLAB 2016a, MathWorks Inc). The frequency domain analysis was done by measuring median frequency of the signal. Fast Fourier Transform (FFT) was performed to the filtered signal to get power density spectrum. Half of total power spectrum was then calculated in order to get median frequency. Muscle fatigue can be indicated by decreasing in median frequency of power spectrum over contraction time (Shi et al., 2007). Decrease in muscle conduction velocity of the motor action potentials at muscle membrane can also cause median frequency to decrease (Medved and Cifrek, 2011; Stirn et al., 2008).

MDF is a frequency value at which the EMG power spectrum is divided into two regions with an equal integrated power. The equation used to calculate Median Frequency is as in Equation (2):

$$
\sum_{j=1}^{M D F} P j=\sum_{j=M D F}^{M} P j=\frac{1}{2} \sum_{j=1}^{M} P j
$$

where $P j$ is the EMG power spectrum at a frequency bin $j$ and $M$ represent the length of frequency bin (Thongpanja et al., 2013).

Further statistical analysis were performed using Microsoft Excel. Pearson Correlation calculation was used to find the relationship between average temperature and temperature difference with median frequency for all the subjects with the significance level $\alpha=0.05$.

The correlation equation used is as shown in Equation (3) where $x$ represents median frequency and $y$ represents either average temperature or temperature difference parameter:

$$
r=\frac{\sum_{i}\left(x_{i}-\bar{x}\right)\left(y_{i}-\bar{y}\right)}{\left.\left.\sqrt{\sum_{i}\left(x_{i}-\bar{x}\right.}\right)^{2} \sqrt{\sum_{i}\left(y_{i}-\bar{y}\right.}\right)^{2}}
$$

\section{RESULTS AND DISCUSSION}

\section{Infrared Thermal imaging}

Exercise for athletes is an activity that required physical effort to be carried out in order to build up their stamina, muscle strength and improve health, fitness and their performance. Fatigue muscle absorbs less energy where it will stretch out to a certain limit that can cause injury. Muscle fatigue usually related to the lack of ability to reach a certain level of maximum voluntary contraction force or reduction in performance of repetitive actions. Localized muscle fatigue can be 
divided into two mechanisms which are central fatigue and peripheral fatigue. Usually, during muscle fatigue it will involve both mechanisms. Central fatigue is related to the central nervous system, which includes connection from brain to the nerves of the contracted muscle during fatigue. When the central nervous system fails to drive the motor neurons adequately due to the changes of various neurotransmitters in the brain this will lead to reduction in nerve-based motor command. Consequently cause the reduction muscles activation which will result in decrease of muscle force output. Peripheral fatigue relates to the ability of muscle to perform physical work. During fatigue, contraction of the muscle is impaired thus muscle's ability to exert force is declines as the body is not able to meet the demand of the increasing energy in the contracting muscle.

There are several factors which can lead to muscle fatigue such as physical, environment, biochemical and nutritional factors. Muscle fatigue can be due to lactic acid-induced muscle fatigue. During exercise, muscle will contract and move the body part. This process of muscle contraction requires oxygen to the cell as it will supply energy for muscle contraction. Adequate oxygen supply makes the cell respiration in aerobic state. However, when the oxygen is getting lesser or no longer available, cells will respirate in anaerobic and waste product of lactic acid will be produced. The increase amount of lactic acid in the muscle tissue and reduction of glycogen will reduce contractile properties of muscle and introduce muscle to pain and fatigue (Al-Mulla et al., 2011). Muscle will also face difficulty in continuing the task. Additionally, muscle fatigue can occur due to deficiency in certain minerals and electrolytes that are necessary for muscle to function properly. Inadequate vital minerals such as potassium, magnesium and calcium will reduce muscle function.

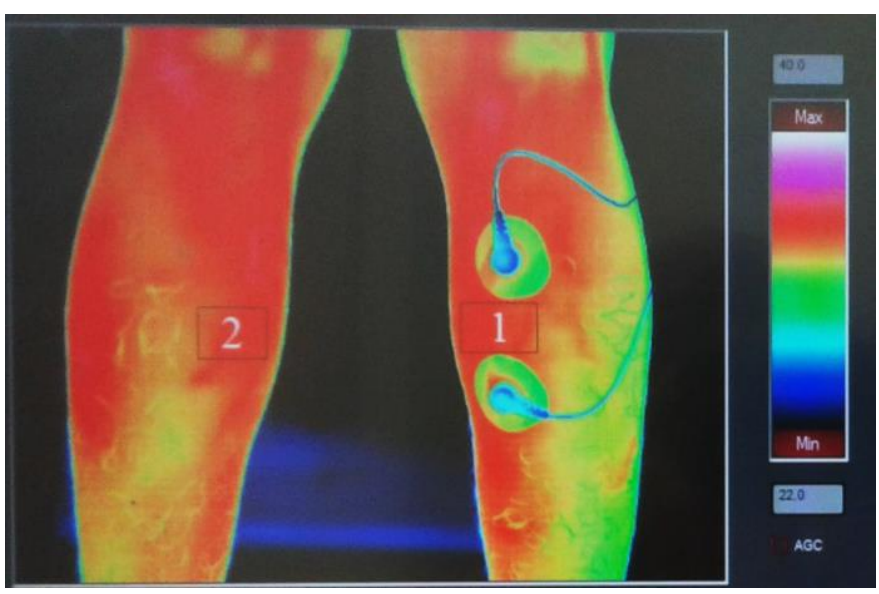

Fig. 3 Thermal imaging image of gastrocnemius muscle before exercise.

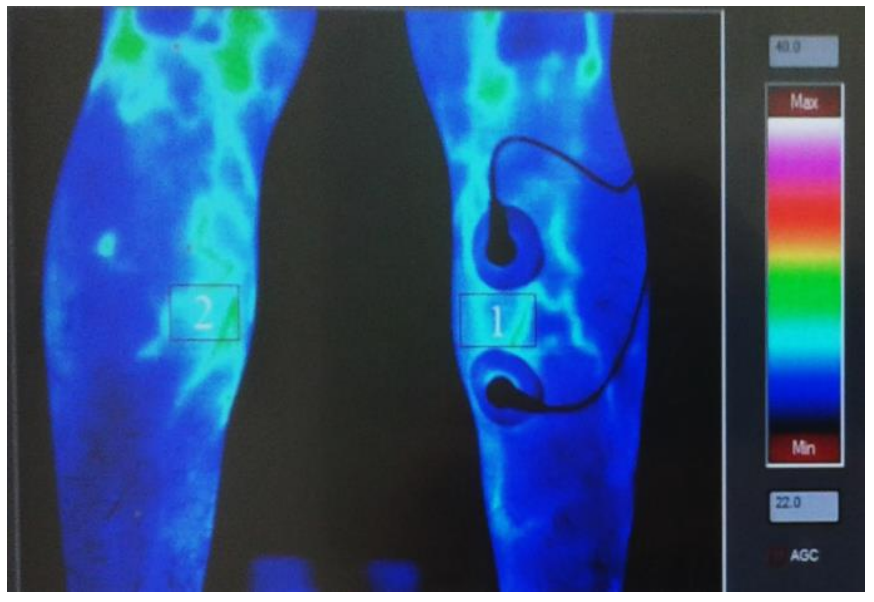

Fig. 4 Thermal imaging image of gastrocnemius muscle during exercise

Figure 3 and 4 show the example of infrared thermal images of gastrocnemius muscle region obtained in two conditions, first before the subjects started with the exercise regime and the second one is after the subject completed the whole exercise regime. Based on the temperature range, subject's thermal image in Figure 3 shows an average temperature of 34.22 degree at the targeted muscle region. As the subject started to run on the treadmill, the overall surface temperature decrease with an average temperature of 26.83 degree at the targeted muscle region. Physiologically, when an athlete starts to perform a physical activity with high intensity, blood needs to be diverted to the area which require more oxygen to generate additional energy (Wallin, 1990). In this particular case, the reduction of temperature was observed as the subject starts to run on the treadmill due to the contraction of blood vessel near the skin surface to divert the large volume of blood to those gastrocnemius tissue muscles.

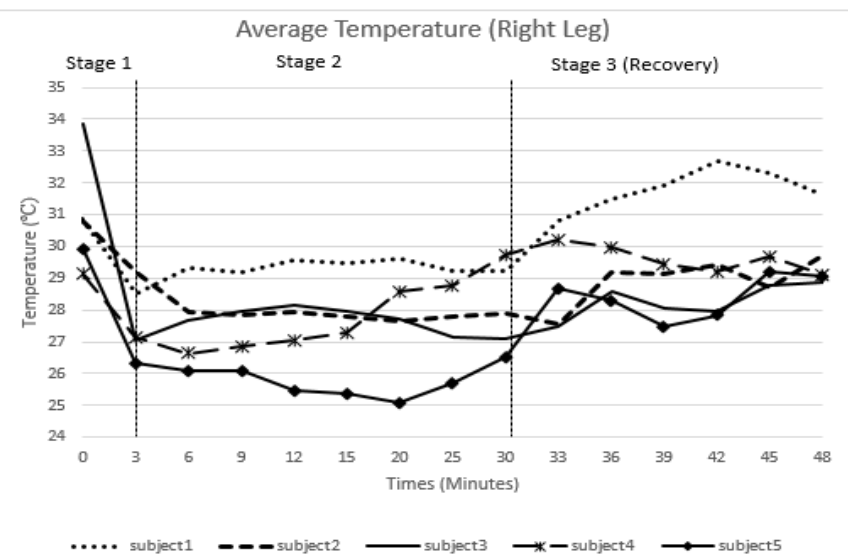

Fig. 5 Average temperature of gastrocnemius muscle between all subjects.

Figure 5 presents the quantitative detail of average skin surface temperature profile within the gastrocnemius muscle region in all subjects' dominant leg before, during and after exercise. An average temperature is estimated by averaging overall values of temperature from a determined regions of interest of gastrocnemius muscle that being recorded using thermal imaging camera. It can be seen that, in the Stage 1, the average temperature decreased dramatically in all subjects. In Stage 2 which is during running progress, the average temperature was observed to retain and fluctuate minimally with respect to the initial basal line. Interestingly, during the recovery period in Stage 3, the average temperature of the targeted area began to increase.

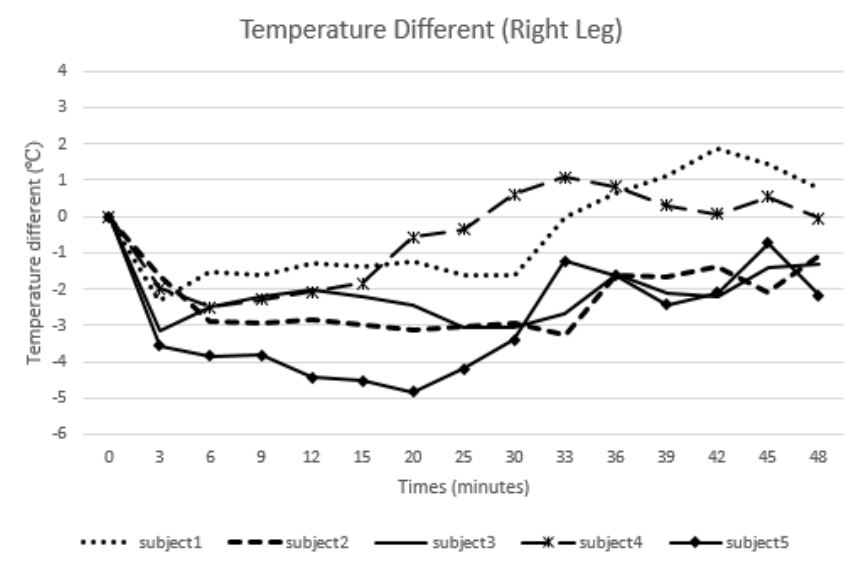

Fig. 6 Temperature difference of gastrocnemius muscle between all subjects.

Figure 6 shows the result of temperature difference of gastrocnemius muscle between all subjects along the exercise. Temperature difference is the difference between current temperatures of the specific time frame with the baseline temperature. 
Result from temperature difference shows total body temperature values were $3-4{ }^{\circ} \mathrm{C}$ lower than at baseline body temperature (Figure 6). This result is correspond with the previous study done by Merla et al. (2010) where they investigated of the cutaneous temperature variations in well-trained runners during graded treadmill exercise until reaching their individual maximal heart rate using thermal imaging. In their study total body cutaneous temperature (Tc) decreased as subjects starts exercise where thighs and forearms exhibit the earliest response. Then further Tc decreased with along the exercise with the values of $3-5^{\circ} \mathrm{C}$ lower than the baseline. During the recovery, Tc is increased where forearms and thighs exhibited the earliest increase.

Exercise will generate heat within the body and there will be thermo regulatory process where human body will regulate the core temperature in order to prevent overheating due heat produced by the contracting muscle. This localized thermal changes of increases and decreases in skin surface temperature can be detected by infrared thermographic marked by variations of temperature (Hildebrandt et al., 2012).

In the beginning of exercise, there is a sudden fall of skin temperature that may due to vasoconstriction of cutaneous vessels for increase the muscular blood flow recruitment of the working muscles (Duc et al., 2015 and Merla et al., 2010). In exercise, muscle contraction demands more oxygen being delivered to the area thus increases blood flow. As the duration of the exercise continued, continuous decrease of skin temperature is observed that may due to core temperature increases the central regulatory mechanism cause vasoconstriction of cutaneous vessels and heat will dissipate through the skin by sweat evaporation. This result is also corresponded by one recent study by Arfaoui et al.(2014) where metabolic heat production increases with increased work intensity thus, raises thermal regulatory processes. This results in a continuous decrease in skin temperature with increase the intensity of exercise.

Thermal images recorded during recovery from exercise showed that increase in skin temperature. This is caused by hyperthermal spots due to the presence of muscle perforator vessels during recovery. The diffusion of heat from the hyperthermal spots to the surrounding cutaneous tissue suggests a possible hemodynamic and thermoregulatory role for the perforator vessels during/after exercise (Merla et al., 2010). The same finding was observed by FernandezCuaves et al. (2014) in their 6 hour post-exercise study. However, many researchers suggested longer time (up to 24 hours) for monitoring muscle recovery and its relation to muscle soreness and inflammatory. Although sweat evaporation could be another factor that contributes to the total heat released which probably captured by thermal imaging camera, Priego Quesada et al. (2015) in their study showed that effect of the perspiration is very minimal.

\section{Electromyography}

Figure 7 presents the value of Median Frequency (MDF) obtained from EMG measurements during the exercise for all subjects. Generally, EMG signal from fatigue muscle is measured within the time or frequency domain (Al-Mulla et al., 2011). In muscle fatigue, MDF indicates the firing rate of action potential in a fatigue muscle. Result in this study shows that MDF values decrease during the exercise. Result obtained in this study is corresponding to a study done by Cardozo et al. (2013) on the behaviour of muscle due to an isometric fatiguing protocol shows that frequency (median frequency) is decrease in fatigue muscle. Another study by Shi et al. (2007) in assessing muscle fatigue on right biceps brachii muscle using sonomyography and EMG for the comparison showed a decrease of MDF as a function of time during the muscle fatigue. The changes in the EMG parameter indicated that the targeted muscles had experienced fatigue during the experiment.

\section{Correlation analysis between infrared thermal imaging and electromyography}

Correlation analysis between the values of MDF which was obtained from EMG measurements during the exercise for all subjects was conducted to assess the relationship between changes in MDF and changes in the temperature of gastrocnemius muscle region for all subjects.

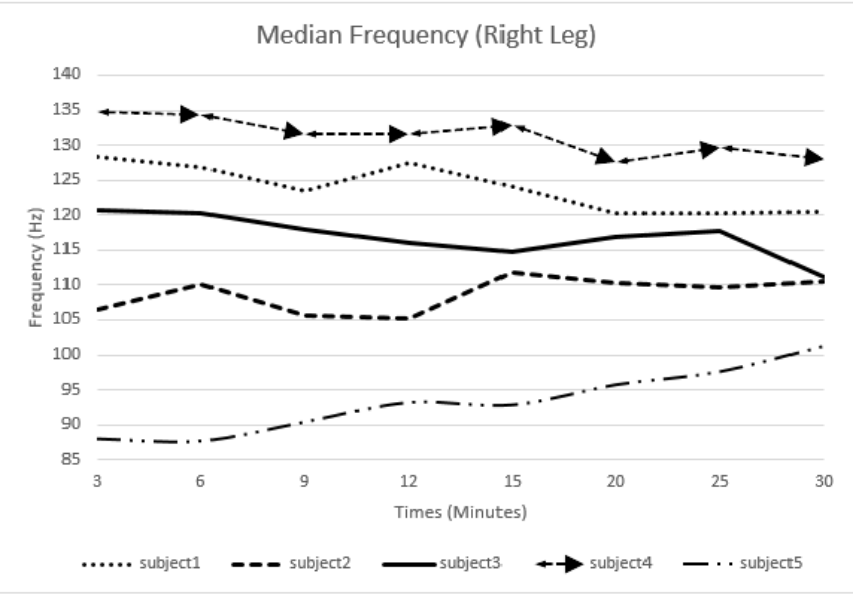

Fig. 7 Median Frequency values of gastrocnemius muscle between all subjects.

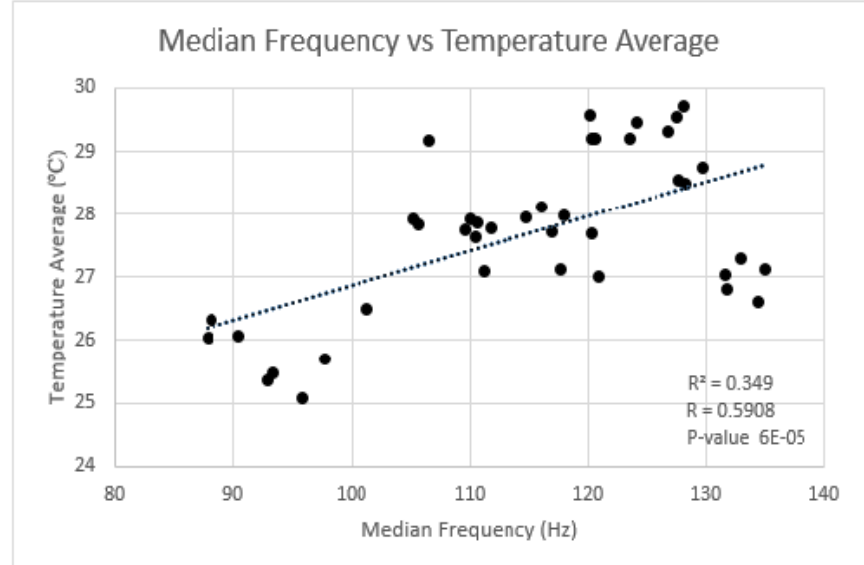

Fig. 8 Correlation between median frequency and temperature average for all subjects.

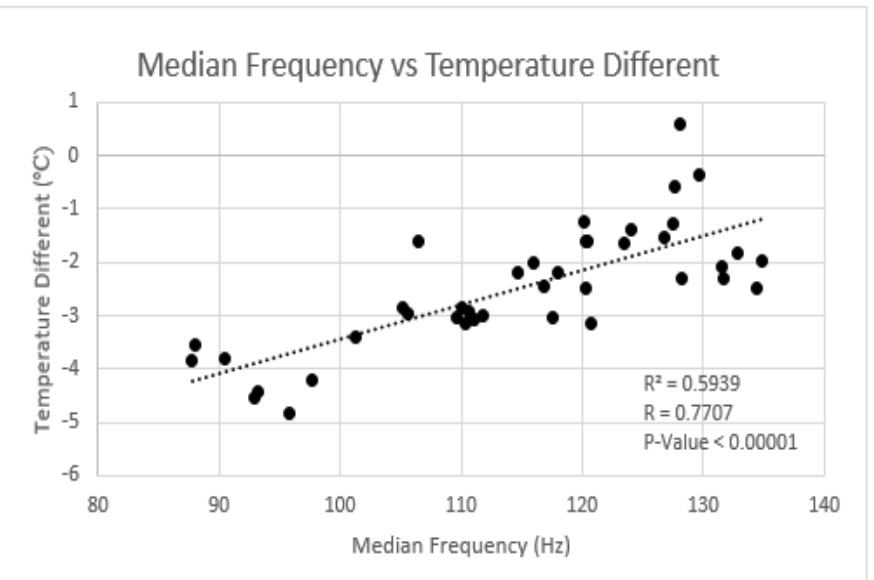

Fig. 9 Correlation between median frequency and temperature difference for all subjects.

Figure 8 shows the correlation plot for between average temperature and MDF parameters, while Figure 9 shows the correlation plot between temperature difference and MDF parameters.

Based on Figure 8, the correlations between average temperature and MDF parameters which were recorded at the same gastrocnemius muscle region shows that there is a significant moderate positive correlation with correlation coefficient value, $R$ of 0.5908 and 
probability of 0.00006 at $\mathrm{p}<0.05$. On the other hand, the correlations between temperature difference and MDF parameters as in Figure 9 shows that there is a significant strong positive correlation with correlation coefficient value, $R$ of 0.7707 with probability $p$, of less than 0.00001 at $\mathrm{p}<0.05$.

Results attained from the correlation analysis show that temperature difference parameter extracted from thermal images is strongly correlated with MDF which corresponded to EMG signal for muscle fatigue. Although it is initially hypothesized that there is a relation between skin surface temperature profile and EMG signal in general, this study however suggested that in order to efficiently evaluate muscle fatigue using thermal imaging technique, temperature difference parameter can be used as one of the indicator for muscle fatigue during dynamic exercise since it show as MDF value reduces, temperature difference between current skin surface and initial skin surface temperatures increases.

Therefore, by using this specific parameter, it will determine an efficient and concise approach in predicting muscle fatigue for further injury prevention, focusing on areas with the highest incidence and severity. This will certainly save a lot of money spent on athletes' medication and rehabilitation. However, more subjects are required in future in order to come out with the specific range of temperature difference as an indicator for muscle fatigue.

\section{CONCLUSION}

Thermal imaging method has shown to be a potential non-invasive tool for muscle fatigue assessment. A series of experiments which were conducted in five healthy men running on a treadmill at a constant speed showed that temperature difference parameter of gastrocnemius muscle surface has a significant strong correlation to median frequency parameter extracted from a standard evaluation method, EMG compared to the average temperature of the skin surface. For future study, other related parameters for muscle fatigue evaluation such as maximal oxygen uptake, heart rate and lactate level can be used together to explore their individual correlation with skin surface temperature profile obtained from infrared thermal imaging camera. Data collection on more subjects should be conducted in order to obtain a standard range of temperature changes in all stages, whereby classification of muscle fatigue can be performed using machine learning technique. As a conclusion, this study has suggested temperature difference parameter to be used as an indicator for muscle fatigue evaluation using infrared thermal imaging.

\section{ACKNOWLEDGEMENT}

Authors would like to express gratitude to Ministry of Higher Education Malaysia and Universiti Teknologi Malaysia for supporting this research under the Institutional Research Grants Vote Number $02 \mathrm{~K} 76$

\section{REFERENCES}

Al-Mulla, M. R., Sepulveda, F. and Colley, M. (2011). A review of non-invasive techniques to detect and predict localised muscle fatigue. Sensors, 11(4), pp. 3545-3594. doi: 10.3390/s110403545

Arfaoui, A., Bertucci, W.M., Letellier, T. and Polidori, G. (2014). Thermoregulation during incremental exercise in masters cycling, Journal of Science and Cycling 3(1), pp. 33-41.

Bartuzi, P., Roman-Liu, D. and Wiśniewski, T. (2012). The influence of fatigue on muscle temperature. International Journal of Occupational Safety and Ergonomics, 18(2), pp. 233-243. doi: 10.1080/10803548.2012.11076931

Cardozo, A.C., Gonçalves M., Halla,C.Z., and Marques, N.R. (2013). Agerelated neuromuscular adjustments assessed by EMG. In Associate Prof. Dr.Hande Turker (Ed.) Electrodiagnosis in New Frontiers of Clinical Research, Croatia: InTech, 2013: 113-29.

Cramer, M. N. and Jay, O. (2016) Biophysical aspects of human thermoregulation during heat stress. Autonomic Neuroscience Basic Clinical 196, pp. 3-13. doi:10.1016/j.autneu.2016.03.001
Duc, S., Arfaoui, A., Polidori, G. and Bertucci, W. (2015). Efficiency and thermography in cycling during a graded exercise test. Journal of Exercise, Sports \& Orthopedics 2 (2): 1-8. Retrieved from https://symbiosisonlinepublishing.com/exercise-sports orthopedics/exercise-sports-orthopedics28.pdf

Fenner, K., Yoon, S., White, P., Starling, M., and McGreevy, P. (2016). The Effect of Noseband Tightening on Horses' Behavior, Eye Temperature, and Cardiac Responses. PLoS ONE 11(5): e0154179. doi:10.1371/journal.pone.0154179

Fernández-Cuevas, I., Sillero-Quintana, M., Garcia-Concepcion, M.A., Serrano J.R., Gomez-Carmona, P., and Marins, J. CB., (2014) Monitoring skin thermal response to training with infrared thermography. New Studies in Athletics 29:57-71.

Formenti, D., Ludwig, N., Trecroci, A., Gargano, M., Michielon, G., Caumo, A., and Alberti, G. (2016). Dynamics of thermographic skin temperature response during squat exercise at two different speeds. Journal of Thermal Biology. 59:58-63. Doi: 10.1016/j. jtherbio.2016.04.013

Gillis, D.J., Barwood, M.J, Newton, P.S., House, J.R., Tipton M.J. (2016). The influence of a menthol and ethanol soaked garment on human temperature regulation and perception during exercise and rest in warm, humid conditions. Journal of Thermal Biology. 58:99-105. doi:10.1016/j.jtherbio.2016.04.009

González-Alonso, J. (2012) Human thermoregulation and the cardiovascular system. Experimental Physiology 97:340-346. doi:10.1113/expphysiol.2011.058701

Hadžić, V., Širok, B., Malneršič, A. and Čoh, M. (2015). In Press, Available online 4 November 2015. Can infrared thermography be used to monitor fatigue during exercise? A case study. Journal of Sport and Health Science, doi: 10.1016/j.jshs.2015.08.002.

Hildebrandt, C., Zeilberger, K., Ring, E. F. J. and Raschner, C. (2012). The application of medical infrared thermography in sports medicine. In Dr. Kenneth R. Zaslav (Ed.), An International Perspective on Topics in Sports Medicine and Sports Injury, (14), pp.257-275. doi: 10.5772/28383

Priego Quesada, J. I. (Ed.) (2017), Application of infrared thermography in Sports Science, Biological and Medical Physics, Biomedical Engineering, Springer. doi: 10.1007/978-3-319-47410-6_1

Kallenberg, L. A. C. and Hermens, H. J. (2008). Behaviour of a surface EMG based measure for motor control: Motor unit action potential rate in relation to force and muscle fatigue. Journal of Electromyography and Kinesiology, 18(5), pp.780-788. doi: 10.1016/j.jelekin.2007.02.011

Lim, C.L., Byrne, C., and Lee, J.K. (2008) Human thermoregulation and measurement of body temperature in exercise and clinical settings. Annals Academy of Medicine Singapore. 37(4):347-353.

Medved, V. and Cifrek, M. (2011). Kinesiological Electromyography, Biomechanics in Applications, Dr Vaclav Klika (Ed.), InTech, DOI: $10.5772 / 21282$. Available https://www.intechopen.com/books/biomechanics-inapplications/kinesiological-electromyography

Merla, A., Mattei, P. A., Di Donato, L. and Romani, G. L. (2010). Thermal imaging of cutaneous temperature modifications in runners during graded exercise. Annals of Biomedical Engineering, 38(1), pp. 158-163. doi: 10.1007/s10439-009-9809-8

Priego Quesada, J. I., Carpes, F.P., Bini, R.R., Salvador, Palmer, R., PérezSoriano P, Cibrián Ortiz de Anda, R.M. (2015). Relationship between skin temperature and muscle activation during incremental cycle exercise. Journal of Thermal Biology. 48:28-35. doi:10.1016/j.jtherbio.2014.12.005

Shi, J., Zheng, Y. P., Chen, X. and Huang, Q. H. (2007). Assessment of muscle fatigue using sonomyography: Muscle thickness change detected from ultrasound images. Medical Engineering and Physics, 29(4), pp.472-479. doi: 10.1016/j.medengphy.2006.07.004

Stirn, I., Jarm, T. and Strojnik, V. (2008). Evaluation of the mean power frequency of the EMG signal power spectrum at endurance levels during fatiguing isometric muscle contractions. Kinesiologia Slovenica, 14(1), pp. 28-38.

Thongpanja, S., Phinyomark, A., Phukpattaranont, P., and Limsakul, C. (2013). Mean and median frequency of EMG signal to determine muscle force based on time-dependent power spectrum. Elektronika IR Elektrotechnika, 19(3), pp. 51-56. doi: 10.5755/j01.eee.19.3.3697

Medved, V. and Cifrek, M. (2011). Kinesiological electromyography. In Klika, V. (Ed.) Biomechanics in Applications, InTech, doi: 10.5772/21282

Merletti, R., Botter, A., Troiano, A., Merlo, E. and Minetto, M. A. (2009) 'Technology and instrumentation for detection and conditioning of the surface electromyographic signal: State of the art', Clinical Biomechanics. Elsevier Ltd, 24(2), pp. 122-134. doi: 10.1016/ j.clinbiomech.2008.08.006.

Wallin, B. G. (1990). Neural control of human skin blood flow. Journal of the Autonomic Nervous System, 30(Suppl.): S185-S190. 